\title{
Heavily T2-Weighted Magnetic Resonance Myelography as a Safe Cerebrospinal Fluid Leakage Detection Modality for Nontraumatic Subdural Hematoma
}

\author{
Sungjae An, ${ }^{1}$ Han-Gil Jeong, ${ }^{1,2}$ Dongwook Seo, ${ }^{1}$ Hyunjun Jo, ${ }^{1, *}$ Si Un Lee, Jae Seung Bang, ${ }^{1}$ Chang Wan Oh, Tackeun Kim ${ }^{1}$ \\ Department of Neurosurgery,' Seoul National University Bundang Hospital, Seoul National University College of Medicine, Seongnam, Korea \\ Department of Neurology, ${ }^{2}$ Seoul National University Bundang Hospital, Seoul National University College of Medicine, Seongnam, Korea
}

Objective : Nontraumatic subdural hematoma (SDH) is a common disease, and spinal cerebrospinal fluid (CSF) leakage is a possible etiology of unknown significance, which is commonly investigated by several invasive studies. This study demonstrates that heavily T2-weighted magnetic resonance myelography (HT2W-MRM) is a safe and clinically effective imaging modality for detecting CSF leakage in patients with nontraumatic SDH.

Methods : All patients who underwent HT2W-MRM for nontraumatic SDH workup at our institution were searched and enrolled in this study. Several parameters were measured and analyzed, including patient demographic data, initial modified Rankin Scale (mRS) score upon presentation, SDH bilaterality, hematoma thickness upon presentation, CSF leakage sites, treatment modalities, followup hematoma thickness, and follow-up mRS score.

Results : Forty patients were identified, of which 22 (55.0\%) had CSF leakage at various spinal locations. Five patients (12.5\%) showed no change in mRS score, whereas the remaining (87.5\%) showed decreases in follow-up mRS scores. In terms of the overall hematoma thickness, four patients (10.0\%) showed increased thickness, two (5.0\%) showed no change, 32 (80.0\%) showed decreased thickness, and two (5.0\%) did not undergo follow-up imaging for hematoma thickness measurement.

Conclusion : HT2W-MRM is not only safe but also clinically effective as a primary diagnostic imaging modality to investigate CSF leakage in patients with nontraumatic SDH. Moreover, this study suggests that CSF leakage is a common etiology for nontraumatic $\mathrm{SDH}$, which warrants changes in the diagnosis and treatment strategies.

Key Words : Hematoma, subdural · Cerebrospinal fluid leak · Intracranial hypotension · Myelography · Magnetic resonance image · Blood patch, epidural.

\section{INTRODUCTION}

Chronic subdural hematoma (SDH) is a well-known disease of elderly individuals as trauma-related intracranial hemorrhage expanded by various mechanisms, including rebleeding, coagulopathy, local inflammation and angiogenesis, exudate

- Received : November 23, 2020 •Revised : January 18, 2021 •Accepted : March 26, 2021

- Address for reprints : Tackeun Kim

Department of Neurosurgery, Seoul National University Bundang Hospital, 82 Gumi-ro 173beon-gil, Bundang-gu, Seongnam 13620, Korea

Tel : +82-31-787-7161, Fax : +82-31-787-4059, E-mail : tackeun.kim@snu.ac.kr, ORCID : https://orcid.org/0000-0002-4375-8095

${ }^{*}$ Current affiliation : Department of Neurosurgery, Korea University Ansan Hospital, Korea University College of Medicine, Ansan, Korea

This is an Open Access article distributed under the terms of the Creative Commons Attribution Non-Commercial License (http://creativecommons.org/licenses/by-nc/4.0) which permits unrestricted non-commercial use, distribution, and reproduction in any medium, provided the original work is properly cited. 
from outer membrane, and cerebrospinal fluid (CSF) entrapment ${ }^{35}$. Minor trauma in patients with SDH could be confirmed by history-taking. However, in the emergency room or outpatient clinics, we occasionally meet patients with SDH without any trauma history, making the determination of the cause of SDH difficult. Those nontraumatic SDH cases represents approximately $50 \%$ of total SDH cases $^{15}$.

Among various possible etiologies for nontraumatic SDH, many studies have investigated the association between spontaneous intracranial hypotension (SIH), a syndrome induced by the leakage of CSF from dural tear and $\mathrm{SDH}^{16}$. Among non-geriatric SDH patients, $25.9 \%$ showed CSF leak by various imaging modalities ${ }^{3)}$. Also, $16-57 \%$ among SIH patients were presented $\mathrm{SDH}^{8,17,27,28,30,36)}$. Considering those strong relationship between SIH and SDH, thorough examination for SIH in SDH patients with unclear etiology should be done to improve patients' outcomes by occlusion of CSF leak ${ }^{11,12,14,22-25,29,34)}$. Although the International Classification of Headache Disorders-3 diagnostic criteria for SIH includes "CSF loss on cranial or spinal imaging or lumbar opening pressure of $<6 \mathrm{cmH}_{2} \mathrm{O}$, spinal imaging to reveal CSF leakage holds high diagnostic value, because the CSF opening pressure is neither sensitive nor specific ${ }^{4}$. Furthermore, accurate evaluation of the CSF leakage site as the target site of injection is mandatory for epidural blood patch (EBP) as a treatment strategy for SIH, which was suggested by several studies ${ }^{5,13}$.

Studies have used invasive modalities to identify CSF leakage sites, such as radionuclide cisternography, computed tomography (CT) myelography, and magnetic resonance myelography (MRM) with contrast agent ${ }^{3,9,30)}$. A universal step in those invasive modalities is lumbar puncture, which could pose a specific risk of CSF leakage through the puncture site, procedure-related infection, and concomitant complications, including $\mathrm{SDH}^{10,21)}$. In addition, complications from radiation or intrathecal contrast injection should be considered ${ }^{18,20}$. Moreover, whether lumbar puncture in patients with preexisting SDH could result in an increased risk of spinal CSF leakage or low intracranial pressure is not fully known.

Heavily T2-weighted MRM (HT2W-MRM) without intrathecal contrast injection is an emerging noninvasive modality to precisely detect CSF leakage sites ${ }^{18)}$. As our institution has recently introduced HT2W-MRM, we have evaluated patients with nontraumatic SDH using this novel modality. Thus, we tried demonstrating the clinical usefulness of HT2W-MRM as a primary diagnostic modality in evaluating suspected $\mathrm{SDH}$ with SIH by investigating cumulative cases. In addition, our study determines how many nontraumatic SDH were related to CSF leakage using HT2W-MRM.

\section{MATERIALS AND METHODS}

This study was approved by the Institutional Review Board of Seoul National University Bundang Hospital (IRB No. B-2008-631-103), and the requirement for informed consent from the patients was waived.

\section{Patients}

Our institution introduced HT2W-MRM in August 2018 as a single primary imaging method for investigating CSF leak$\operatorname{age}^{18}$. Patients who were unfit for magnetic resonance imaging (MRI) or those with poor MRI acquisition quality due to cooperation problems underwent CT myelography. Therefore, almost all patients suspected of SIH or SIH-related SDH since August 2018 in our institution had been screened using HT2WMRM.

We reviewed our institution's electronic medical records and identified 73 patients who 1) had undergone HT2WMRM and simultaneously 2) had been diagnosed with SDH using any diagnostic imaging modality. Among the 73 identified patients, 33 patients who had an obvious etiology of SDH other than CSF leakage, such as head trauma, any cranial or spinal surgeries within 6 months, and any hematologic disorders, were excluded. Finally, 40 patients with nontraumatic SDH who underwent HT2W-MRM to determine CSF leakage or SIH were enrolled in this study.

Several parameters were described regarding the patients' initial presentation. The patients' overall neurological status was defined using the modified Rankin Scale (mRS) and categorized as independent (for mRS grades 0-2) and dependent (for mRS grades 3-5). SDH location (bilaterality) and maximal thickness were measured using the initial CT or MRI upon the first presentation. CSF leakage sites were specified using HT2W-MRM and categorized as single/multiple and cervical/thoracic/lumbar.

\section{HT2W-MRM}

HT2W-MRM is mostly prescribed and used in two situa- 
tions in Seoul National University Bundang Hospital. First, when SIH is clinically suspected from neurology outpatient clinic or emergency department visit, HT2W-MRM is performed as the primary investigation procedure. Second, for patients diagnosed with SDH, if CSF leakage is suspected as a probable cause, HT2W-MRM is prescribed by neurosurgeons.

Technically, the machine used to perform HT2W-MRM is a 3.0 T MR unit with a routine protocol of heavily T2-weighted sagittal, fat-suppressed three-dimensional (3D) fast spinecho pulse sequence. Two sequences of sagittal 3D images were separately acquired for the cranial and caudal spines; then, multiplanar reformation was performed for axial and coronal images $^{18)}$.

\section{Treatment and outcomes}

Whenever CSF leakage is revealed on MRI, fluoroscopyguided EBP is performed by interventional radiologists on a referral basis. The EBP procedure uses a mixture of autologous blood and $1 \mathrm{~mL}$ of contrast agent (Omnipaque 300; GE Healthcare, Boston, MA, USA) at a maximum of $10 \mathrm{~mL}$ per injection. Targeted EBP procedures are performed after confirming one or more CSF leakage levels using HT2W-MRM, either at the specific CSF leakage level, at the center of the segmental leak, or at two sites to sufficiently cover diffuse or multifocal leaks ${ }^{18)}$.

Hematoma removal operation, EBP, middle meningeal artery embolization (MMAE), or a combination of these procedures was decided by the attending neurosurgeons ${ }^{2,19)}$. To assess the outcomes at the latest follow-up visit within 6 months, $\mathrm{mRS}$ and maximal thickness of hematoma were measured.

The SDH resolution was defined as the maximum followup hematoma thickness of less than $5 \mathrm{~mm}$. A favorable clinical outcome was defined as a follow-up mRS grade of $0-2$.

\section{Statistical analysis}

Statistical analyses were performed using R (version 4.0.0; open-source software, www.r-project.org). All categorical variables were presented as raw data. All numerical continuous variables were presented as mean \pm standard deviation. SDH thickness was measured in millimeters.

\section{RESULTS}

The mean age of the 40 enrolled patients was $62.9 \pm 15.0$ years, of which $31(77.5 \%)$ were male. The $95 \%$ confidence interval for the proportion of males was $61.5-89.2 \%$, indicating statistically significant male predominance. Nineteen patients $(47.5 \%)$ were categorized as independent (initial mRS grade of $0-2)$, whereas the remaining 21 (52.5\%) belonged to the dependent (initial mRS grade of 3-5) group. Eleven patients (27.5\%) showed unilateral SDH with a mean maximal SDH thickness of $17.45 \pm 8.41 \mathrm{~mm}$, whereas $29(72.5 \%)$ showed bilateral SDH and had a mean maximal SDH thickness of $25.6 \pm$ $13.5 \mathrm{~mm}$. Eighteen (45.0\%) had no CSF leakage on HT2WMRM. The remaining patients $(\mathrm{n}=22,55.0 \%)$ had various $\mathrm{CSF}$ leakage sites ranging from single to diffuse spinal level. Among the 22 patients with CSF leakage, six had single leakage lesions (three, two, and one for cervical, thoracic, and lumbar sites, respectively), and 16 had multiple leakage lesions (one, five, two, two, and six for cervical, cervicothoracic, thoracic, thoracolumbar, and cervicothoracolumbar sites, respectively). Patient characteristics are summarized in Table 1, and SDH thickness measurements are summarized in Table 2.

Among the 22 and 18 patients with and without CSF leakage, $16(72.7 \%)$ and $13(72.2 \%)$ presented bilateral SDH, respectively. The chi-square test did not show statistical differences between CSF leakage and bilaterality ( $p=0.97$ ), between CSF leakage and sex $(p=0.476)$, between CSF leakage and initial mRS ( $p=0.356$ ), and between CSF leakage and follow-up mRS ( $p=1.000)$. Lastly, independent t-test did not show a statistical difference between CSF leakage and age $(p=0.221)$.

Among the 22 patients with CSF leakage, 10 underwent both hematoma removal operation and EBP (Fig. 1) with resolution of SDH for six patients (60.0\%) and favorable clinical outcomes for most $(n=9,90.0 \%)$. Nine patients had EBP, only including one patient who had a follow-up clinic visit without an imaging study. Among the nine patients who underwent EBP, four (44.4\%) showed SDH resolution, and all (100.0\%) had favorable clinical outcomes. One patient underwent all three treatment modalities (hematoma removal operation, EBP, and MMAE) and showed a decreased amount of SDH that did not reach resolution. However, follow-up clinical evaluation revealed favorable clinical outcomes. Another patient underwent both EBP and MMAE and showed both SDH resolution and favorable clinical outcomes. Lastly, for another 
Table 1. Patient characteristics

\begin{tabular}{|c|c|c|c|c|}
\hline & Total $(n=40)$ & CSF leakage $(+)(n=22)$ & CSF leakage $(-)(n=18)$ & $p$-value \\
\hline Age (years) & $62.9 \pm 15.0$ & $65.6 \pm 12.7$ & $59.6 \pm 17.2$ & 0.221 \\
\hline Sex, male & $31(77.5)$ & $16(72.7)$ & $15(83.3)$ & 0.476 \\
\hline Initial mRS & & & & 0.356 \\
\hline Independent, $\leq 2$ & $19(47.5)$ & $9(40.9)$ & $10(55.6)$ & \\
\hline Dependent, $>2$ & $21(52.5)$ & $13(59.1)$ & $8(44.4)$ & \\
\hline Bilateral SDH & $29(72.5)$ & $16(72.7)$ & $13(72.2)$ & 0.945 \\
\hline \multicolumn{5}{|l|}{ CSF leakage } \\
\hline Single cervical & $3(7.5)$ & $3(13.6)$ & & \\
\hline Single thoracic & $2(5.0)$ & $2(9.1)$ & & \\
\hline Single lumbar & $1(2.5)$ & $1(4.5)$ & & \\
\hline Multiple cervical & $1(2.5)$ & $1(4.5)$ & & \\
\hline Multiple cervico-thoracic & $5(12.5)$ & $5(22.7)$ & & \\
\hline Multiple thoracic & $2(5.0)$ & $2(9.1)$ & & \\
\hline Multiple thoraco-lumbar & $2(5.0)$ & $2(9.1)$ & & \\
\hline Multiple cervico-thoraco-lumbar & $6(15.0)$ & $6(27.3)$ & & \\
\hline \multicolumn{5}{|l|}{ Treatment modalities } \\
\hline Hematoma removal operation & $22(55.0)$ & $11(50.0)$ & $11(61.1)$ & \\
\hline EBP & $23(57.5)$ & $21(95.5)$ & $2(11.1)$ & \\
\hline MMAE & $3(7.5)$ & $2(9.1)$ & $1(5.6)$ & \\
\hline Follow up mRS & & & & 1.000 \\
\hline Favorable, $\leq 2$ & $38(95.0)$ & $21(95.5)$ & $17(94.4)$ & \\
\hline Poor, $>2$ & $2(5.0)$ & $1(4.5)$ & $1(5.6)$ & \\
\hline
\end{tabular}

Values are presented as mean \pm standard deviation or number (\%). CSF : cerebrospinal fluid, mRS : modified Rankin Scale, SDH : subdural hemorrhage, EBP : epidural blood patch, MMAE : middle meningeal artery embolization

Table 2. SDH thickness measurements

\begin{tabular}{lccc}
\hline & Total $(\mathbf{n}=\mathbf{4 0})$ & CSF leakage $(+)(\mathbf{n}=\mathbf{2 2})$ & CSF leakage $(-)(\mathbf{n}=\mathbf{1 8})$ \\
\hline Unilateral SDH & 11 & 6 & 5 \\
Initial $(\mathrm{mm})$ & $17.5 \pm 8.41$ & $17.7 \pm 7.74$ & $17.2 \pm 10.1$ \\
Follow up $(\mathrm{mm})$ & $6.2 \pm 9.37$ & $10.2 \pm 11.5$ & $1.6 \pm 2.3$ \\
Change $(\mathrm{mm})$ & $-11.2 \pm 10.9$ & $-7.5 \pm 11.5$ & $-15.6 \pm 9.24$ \\
Bilateral SDH & 29 & 16 & 13 \\
Initial (mm) & $25.6 \pm 13.5$ & $25.7 \pm 16.4$ & $25.5 \pm 9.39$ \\
Follow up (mm) & $11.8 \pm 11.6^{*}$ & $9.47 \pm 12.4^{\dagger}$ & $14.7 \pm 10.2^{\ddagger}$ \\
Change $(\mathrm{mm})$ & $-15.1 \pm 11.2^{*}$ & $-17.5 \pm 10.8^{\dagger}$ & $-12.2 \pm 11.3^{\ddagger}$ \\
\hline
\end{tabular}

Values are presented as mean \pm standard deviation. * Measured for 27 patients except two patients without follow up imaging. ${ }^{\dagger}$ Measured for 15 patients except one patient without follow up imaging. ${ }^{\ddagger}$ Measured for 12 patients except one patient without follow up imaging. SDH : subdural hemorrhage, CSF : cerebrospinal fluid

patient, the wait-and-see strategy was used and the patient showed both SDH resolution and favorable clinical outcomes.
Among the 18 patients who did not demonstrate CSF leakage on HT2W-MRM, 10 underwent hematoma removal oper- 


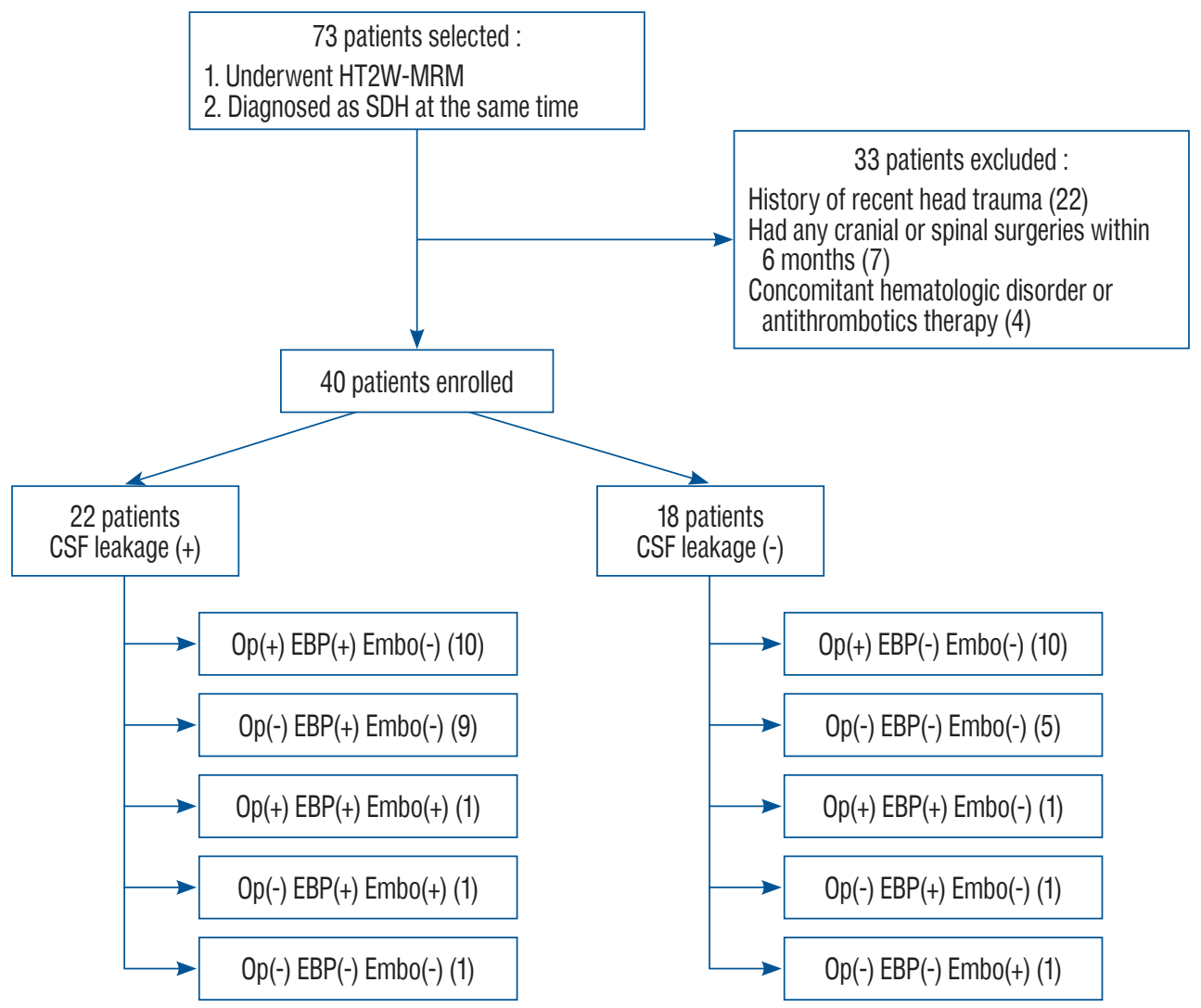

Fig. 1. Patient selection and subgrouping flowchart. Number inside bracket represents patient count. HT2W-MRM : heavily T2-weighted magnetic resonance myelography, SDH : subdural hematoma, CSF : cerebrospinal fluid, Op : hematoma removal operation, EBP : epidural blood patch, Embo : middle meningeal artery embolization.

ation : only six $(60.0 \%)$ had SDH resolution and nine $(90.0 \%)$ had favorable clinical outcomes. For five patients, the waitand-see strategy was used, including one patient who had a follow-up clinic visit without undergoing an imaging study. Two (40.0\%) of them showed SDH resolution, and all of them (100.0\%) had favorable clinical outcomes. One patient underwent MMAE only and showed favorable clinical outcomes, but no SDH resolution. Two patients underwent empirical EBP at the suspicious level under clinical correlation of symptoms. One patient underwent both hematoma removal operation and empirical EBP and showed both SDH resolution and favorable clinical outcomes. One patient underwent empirical EBP only and showed favorable clinical outcomes, but no SDH resolution.

Among all patients in this study, except for two who did not undergo a follow-up imaging study, four (10.0\%) showed an increase in hematoma thickness. Two of them had demonstrated CSF leakage on HT2W-MRM at the initial presentation and necessitated further treatment due to an increase in hematoma size despite undergoing EBP with or without hematoma removal operation. Furthermore, two of them showed no CSF leakage on HT2W-MRM, but hematoma size increased following hematoma removal operation and conservative management, followed by readmission and burr hole surgery.

Among all patients in this study, five (12.5\%) showed no changes in mRS grade, with one having $\mathrm{mRS}$ grade 4 , one having mRS grade 0 , and three having $\mathrm{mRS}$ grade 1 (Fig. 2). Thirty-five patients (87.5\%) showed a decrease in mRS grade at follow-up by $1-4$ grades.

\section{DISCUSSION}

Neurosurgeons commonly encounter SDH in the clinical setting, and treatment outcomes are not always favorable due to little advancement in the treatment modalities for SDH. Although socioeconomic factors would influence the treatment 

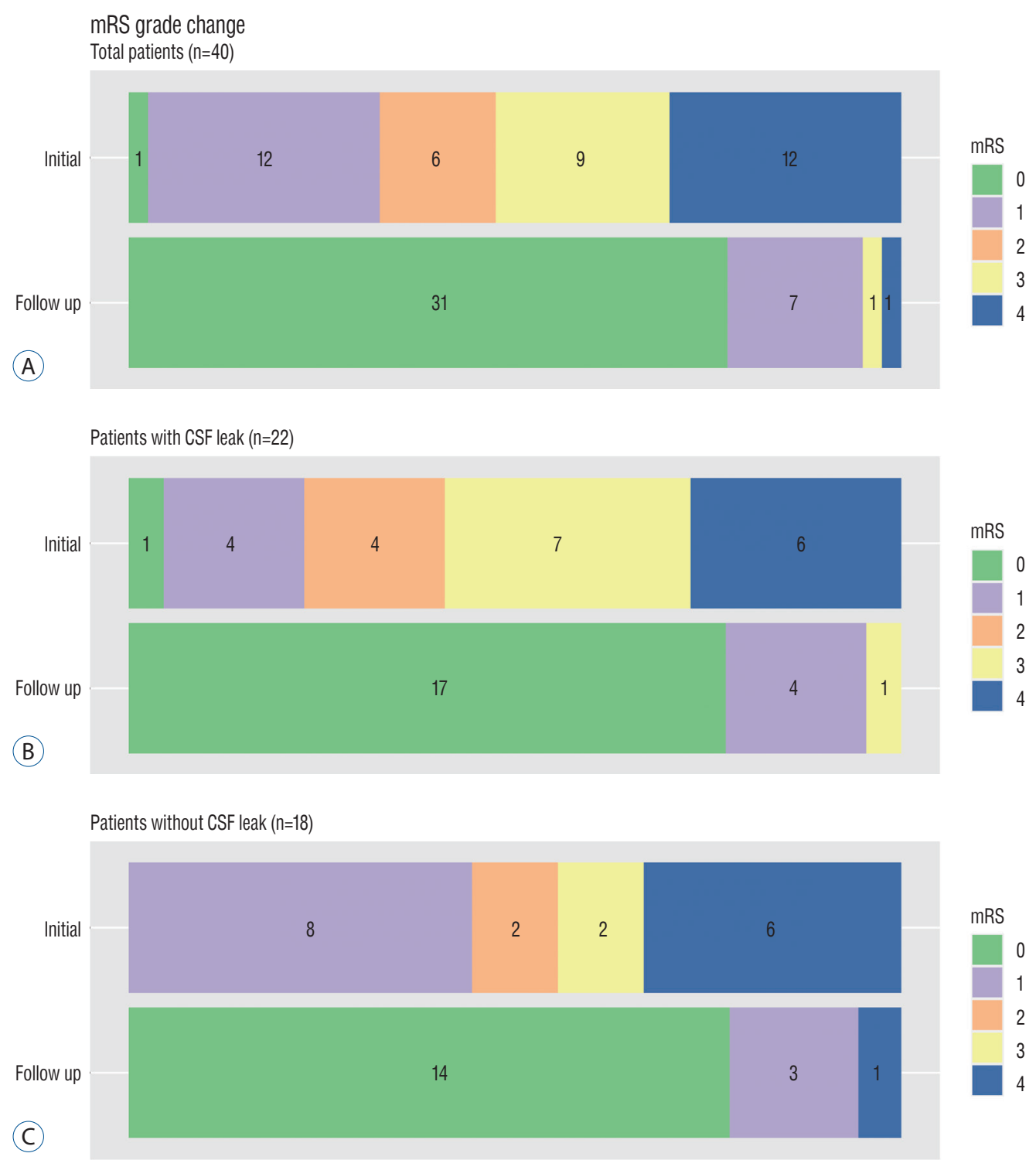

Fig. 2. A-C : Stacked bar chart for mRS grade change from initial presentation to the latest follow up visit within 6 months. Graph legend on the right side indicates bar colors assigned to each mRS grades. Each number at the center of the bar indicates patient count for specific mRS grades. $\mathrm{mRS}$ : modified Rankin Scale.

course and outcomes of patients with SDH, multiple studies have demonstrated a sizable percentage of mortality (3-8\%) and morbidity $(5-12 \%)^{1)}$, which should have room for improvement.

This study's primary focus was nontraumatic SDH possibly caused by SIH, as our concern was that $\mathrm{SIH}$ as a cause of SDH is underestimated and undertreated. SIH prevalence in USA is supposedly 5 per 100000 according to one observational study $^{26)}$, however the prevalence of SIH among nontraumatic $\mathrm{SDH}$ patients is not being investigated. Furthermore, from a few studies ${ }^{3,79,30)}$, notably, clinical suspicion and CSF leakage detection is a crucial step as EBP cannot be performed without confirmation.

Previously, CSF leakage was evaluated using a few imaging modalities, such as MR myelography, CT myelography, and radioisotope scintigraphy, with CT myelography as the current gold standard ${ }^{4}$. Due to the invasiveness of these imaging modalities, we focused on HT2W-MRM, the quality of which is known to be comparable to CT myelography regarding the CSF leakage detection rate for patients with $\mathrm{SIH}^{18,31-33)}$. In ad- 
dition, HT2W-MRM is free from concerns on rare but serious complications. Intrathecal contrast injection could elicit seizures in both patients with and without epilepsy ${ }^{20)}$ and those with intracranial hypotension or $\mathrm{SDH}^{10,21)}$, and eliminating additional morbidity and mortality at the diagnosis stage is worth trying. Radiation is another factor to be concerned about, and HT2W-MRM can be performed with fewer personnel due to the ease of procedure. For patients in this study, HT2W-MRM was performed as the primary diagnostic method for CSF leakage, with a favorable and superior clinical outcome compared with other imaging modalities ${ }^{6}$, and this outcome is consistent in patients with or without CSF leakage on HT2W-MRM (Fig. 2).

In addition, our data showed another important finding that 55\% of patients had CSF leakage, suggesting that CSF leakage might be underestimated as a cause of nontraumatic $\mathrm{SDH}$ and requires good clinical suspicion of neurosurgeons at the diagnosis stage. It is undeniable that selection bias might play a role for patients with poor general condition which precludes transfer for MRI. However, besides those rare cases, as high as $55.0 \%$ of CSF-leakage detection among our study subjects possibly stems from increased application of HT2WMRM at our institution, as all nontraumatic cases with even small possibility of SIH underwent HT2W-MRM. Further study is warranted to reveal the percentage of patients with CSF leakage among patients with nontraumatic SDH, for which current study is not specifically designed to prove. Therefore, we suggest using HT2W-MRM early as the primary CSF leakage detection modality in patients with nontraumatic SDH to further change the diagnosis and treatment strategies.

This study has few limitations. First, HT2W-MRM is not the gold standard in the diagnosis of CSF leakage site due to its innate nature. HT2W-MRM only detects the already extravasated CSF in the epidural space, which might not correspond to active leakage points as the CSF can travel throughout the epidural space. Thus, although the clinical outcomes of using HT2W-MRM were favorable in this study, dynamic imaging modalities, such as dynamic myelography and digital subtraction myelography, could be necessary. Second, as the decision to start or the timing of the HT2W-MRM study is dependent solely on the physician without an established protocol, not all patients with nontraumatic SDH possibly caused by CSF leakage were included in the study. Further study eliminating this selection bias is warranted.

\section{CONCLUSION}

HT2W-MRM is a safe and effective diagnostic modality for CSF leakage in patients with nontraumatic SDH. Furthermore, CSF leakage could be a common etiology of nontraumatic SDH than our knowledge, which warrants further investigations.

\section{CONFLICTS OF INTEREST}

No potential conflict of interest relevant to this article was reported.

\section{INFORMED CONSENT}

This type of study does not require informed consent.

\section{AUTHOR CONTRIBUTIONS}

\author{
Conceptualization : SA, TK \\ Data curation : SA \\ Methodology: SA, TK \\ Project administration : TK \\ Visualization : SA \\ Writing - original draft : SA \\ Writing - review \& editing: TK, HGJ, DS, HJ, SUL, JSB, \\ $\mathrm{CWO}$
}

\section{ORCID}

$\begin{array}{ll}\text { Sungjae An } & \text { https://orcid.org/0000-0003-2033-6049 } \\ \text { Han-Gil Jeong } & \text { https://orcid.org/0000-0002-1418-1768 } \\ \text { Dongwook Seo } & \text { https://orcid.org/0000-0003-4165-8178 } \\ \text { Hyunjun Jo } & \text { https://orcid.org/0000-0002-6917-2201 } \\ \text { Si Un Lee } & \text { https://orcid.org/0000-0001-5788-2014 } \\ \text { Jae Seung Bang } & \text { https://orcid.org/0000-0001-9543-8758 } \\ \text { Chang Wan Oh } & \text { https://orcid.org/0000-0001-7311-7072 }\end{array}$


Tackeun Kim https://orcid.org/0000-0002-4375-8095

\section{References}

1. Almenawer SA, Farrokhyar F, Hong C, Alhazzani W, Manoranjan B, Yarascavitch $B$, et al. : Chronic subdural hematoma management: a systematic review and meta-analysis of 34,829 patients. Ann Surg 259 : 449-457, 2014

2. Ban SP, Hwang G, Byoun HS, Kim T, Lee SU, Bang JS, et al. : Middle meningeal artery embolization for chronic subdural hematoma. Radiology 286 : 992-999, 2018

3. Beck J, Gralla J, Fung C, Ulrich CT, Schucht P, Fichtner J, et al. : Spinal cerebrospinal fluid leak as the cause of chronic subdural hematomas in nongeriatric patients. J Neurosurg 121 : 1380-1387, 2014

4. Beck J, Häni L, Ulrich CT, Fung C, Jesse CM, Piechowiak E, et al. : Diagnostic challenges and therapeutic possibilities in spontaneous intracranial hypotension. Clin TransI Neurosci 2 : 2514183X18787371, 2018

5. Berroir S, Loisel B, Ducros A, Boukobza M, Tzourio C, Valade D, et al. : Early epidural blood patch in spontaneous intracranial hypotension. Neurology 63 : 1950-1951, 2004

6. Busl KM, Prabhakaran S : Predictors of mortality in nontraumatic subdural hematoma. J Neurosurg 119 : 1296-1301, 2013

7. Chen $\mathrm{HH}$, Huang $\mathrm{Cl}$, Hseu SS, Lirng JF : Bilateral subdural hematomas caused by spontaneous intracranial hypotension. J Chin Med Assoc 71 : 147-151, 2008

8. Chen YC, Wang YF, Li JY, Chen SP, Lirng JF, Hseu SS, et al. : Treatment and prognosis of subdural hematoma in patients with spontaneous intracranial hypotension. Cephalalgia 36 : 225-231, 2016

9. Chung SJ, Lee JH, Kim SJ, Kwun BD, Lee MC : Subdural hematoma in spontaneous CSF hypovolemia. Neurology 67 : 1088-1089, 2006

10. Dehaene S, Biesemans J, Van Boxem K, Vidts W, Sterken J, Van Zundert $\mathrm{J}$ : Post-dural puncture headache evolving to a subdural hematoma: a case report. Pain Pract $21: 83-87,2021$

11. Dhillon AK, Rabinstein AA, Wijdicks EF : Coma from worsening spontaneous intracranial hypotension after subdural hematoma evacuation. Neurocrit Care 12 : 390-394, 2010

12. Ferrante E, Arpino I, Citterio A, Savino A : Coma resulting from spontaneous intracranial hypotension treated with the epidural blood patch in the trendelenburg position pre-medicated with acetazolamide. Clin Neurol Neurosurg $111: 699-702,2009$

13. Ferrante $E$, Olgiati $E$, Sangalli V, Rubino F : Early pain relief from orthostatic headache and hearing changes in spontaneous intracranial hypotension after epidural blood patch. Acta Neurol Belg 116 : 503-508, 2016

14. Ferrante E, Trimboli M, Pontrelli G, Rubino F : Early coma awakening after epidural blood patch. J Clin Neurosci 71 : 295-296, 2020

15. Frontera JA, Egorova N, Moskowitz AJ : National trend in prevalence, cost, and discharge disposition after subdural hematoma from 1998-

2007. Crit Care Med 39 : 1619-1625, 2011

16. Gordon N : Spontaneous intracranial hypotension. Dev Med Child
Neurol 51 : 932-935, 2009

17. Hashizume K, Watanabe K, Kawaguchi M, Fujiwara A, Furuya H : Evaluation on a clinical course of subdural hematoma in patients undergoing epidural blood patch for spontaneous cerebrospinal fluid leak. Clin Neurol Neurosurg 115 : 1403-1406, 2013

18. Kim BR, Lee JW, Lee E, Kang Y, Ahn JM, Kang HS : Utility of heavily T2weighted MR myelography as the first step in CSF leak detection and the planning of epidural blood patches. J Clin Neurosci 77 : 110-115, 2020

19. Kim J, Moon J, Kim T, Ahn S, Hwang G, Bang J, et al. : Risk factor analysis for the recurrence of chronic subdural hematoma: a review of 368 consecutive surgical cases. Korean J Neurotrauma 11 : 63-69, 2015

20. Klein KM, Shiratori K, Knake S, Hamer HM, Fritsch B, Todorova-Rudolph $A$, et al. : Status epilepticus and seizures induced by iopamidol myelography. Seizure $13: 196-199,2004$

21. Louhab N, Adali N, Laghmari M, Hymer WE, Ben Ali SA, Kissani N : Misdiagnosed spontaneous intracranial hypotension complicated by subdural hematoma following lumbar puncture. Int J Gen Med 7 : 71 73, 2014

22. Mao YT, Dong Q, Fu JH : Delayed subdural hematoma and cerebral venous thrombosis in a patient with spontaneous intracranial hypotension. Neurol Sci 32 : 981-983, 2011

23. Perez-Vega C, Robles-Lomelin P, Robles-Lomelin I, Diaz-Alba A, Navarro VG : Acute subdural hematoma recurrence during drain removal associated with spontaneous intracranial hypotension - a non-reported complication. Surg Neurol Int $11: 316,2020$

24. Sayer FT, Bodelsson M, Larsson EM, Romner B : Spontaneous intracranial hypotension resulting in coma: case report. Neurosurgery 59 : E204; discussion E204, 2006

25. Schievink WI : Stroke and death due to spontaneous intracranial hypotension. Neurocrit Care 18 : 248-251, 2013

26. Schievink WI, Maya MM, Moser F, Tourje J, Torbati S : Frequency of spontaneous intracranial hypotension in the emergency department. J Headache Pain 8 : 325-328, 2007

27. Schievink WI, Maya MM, Moser FG, Tourje J : Spectrum of subdural fluid collections in spontaneous intracranial hypotension. J Neurosurg 103 : 608-613, 2005

28. Schievink WI, Maya MM, Pikul BK, Louy C : Spontaneous spinal cerebrospinal fluid leaks as the cause of subdural hematomas in elderly patients on anticoagulation. J Neurosurg 112 : 295-299, 2010

29. Souirti Z, Benzagmout M, Belahsen F, Chaoui ME : Spontaneous bilateral subacute subdural hematoma revealing intracranial hypotension. Neurosciences (Riyadh) $14:$ 384-385, 2009

30. Takahashi K, Mima T, Akiba Y : Chronic subdural hematoma associated with spontaneous intracranial hypotension: therapeutic strategies and outcomes of 55 cases. Neurol Med Chir (Tokyo) 56 : 69-76, 2016

31. Tsai PH, Fuh JL, Lirng JF, Wang SJ : Heavily T2-weighted MR myelography in patients with spontaneous intracranial hypotension: a casecontrol study. Cephalalgia 27 : 929-934, 2007

32. Tsai PH, Fuh JL, Lirng JF, Wang SJ : Comparisons between heavily T2weighted MR and CT myelography studies in two patients with sponta- 
T2 MR Myelography and Nontraumatic SDH | An S, et al.

neous intracranial hypotension. Cephalalgia 28 : 653-657, 2008

33. Wang YF, Lirng JF, Fuh JL, Hseu SS, Wang SJ : Heavily T2-weighted MR myelography vs $\mathrm{CT}$ myelography in spontaneous intracranial hypotension. Neurology 73 : 1892-1898, 2009

34. Whiteley W, Al-Shahi R, Myles L, Lueck CJ : Spontaneous intracranial hypotension causing confusion and coma: a headache for the neurologist and the neurosurgeon. Br J Neurosurg 17 : 456-458, 2003
35. Yadav YR, Parihar V, Namdev H, Bajaj J : Chronic subdural hematoma. Asian J Neurosurg $11:$ 330-342, 2016.

36. Yoon SH, Chung YS, Yoon BW, Kim JE, Paek SH, Kim DG : Clinical experiences with spontaneous intracranial hypotension: a proposal of a diagnostic approach and treatment. Clin Neurol Neurosurg 113 : 373-379, 2011 